\title{
Normalization of P-wave Axis After CTI Ablation
}

\author{
Parth Makker ${ }^{1}$, Eric Dulmovits ${ }^{2}$, and Stuart Beldner ${ }^{3}$ \\ ${ }^{1}$ Lenox Hill Hospital \\ ${ }^{2}$ North Shore University Hospital \\ ${ }^{3}$ Northwell Health
}

October 20, 2020

\begin{abstract}
We report a case of superiorly directed sinus p-waves in a young patient who had extensive right atrial scarring due to prior right atriotomies. This sinus p-wave axis then changed to inferiorly directed after catheter ablation of the cavo-tricuspid isthmus (CTI). Normal p-wave morphology during sinus rhythm has been described as having an axis between 0 to +75 degrees. However, this is contingent upon an otherwise healthy atrium without conduction abnormalities. Our case highlights a young patient who has undergone two mitral valve surgeries, and as a result, two right atriotomies for cardiopulmonary bypass. During follow-up after the second surgery, his unusual p-wave axis was noted and it was unclear whether it was due to an atrial escape rhythm that had overtaken the sinus node or abnormal conduction of sinus rhythm. Electroanatomic activation mapping proved that he indeed had a sinus rhythm with conduction abnormality along the sites of his previous atriotomies. Additional ablation on the other side of the sinus node exit resulted in pseudo-normalization of his p-wave axis morphology. Four week follow up showed persistent inferiorly directed $\mathrm{P}$ wave, a finding in this patient consistent with persistent block across the caval tricuspid isthmus.
\end{abstract}

\section{Normalization of P-wave Axis After CTI Ablation}

Parth Makker MD ${ }^{1}$, Eric Dulmovits MD ${ }^{1}$, Stuart Beldner MD FHRS ${ }^{1}$

Northwell Health - 300 Community Dr, Manhasset, NY 11030

Corresponding Author:

Parth Makker

11 East $12^{\text {th }}$ St, Apt $1 \mathrm{~F}$

New York, NY 10003

The authors have no conflicts of interest to disclose

The authors did not receive funding for this case report

Word Count: 569 words

Introduction:

Electrocardiographic (ECG) criteria for p-wave morphology in sinus rhythm requires a left-inferior axis $(0.0$ to +75 degrees). ${ }^{1}$ However, the normal p-wave morphology can be altered by physiologic or post-surgical changes in the right or left atrium due to enlargement of the atria or scarring. ${ }^{1,2}$ 
We report a case of superiorly directed sinus p-waves in a young patient who had extensive right atrial scarring due to prior right atriotomies. This sinus p-wave axis then changed to inferiorly directed after catheter ablation of the cavo-tricuspid isthmus (CTI).

\section{Case:}

Our patient is a 32-year-old man with a history of severe mitral regurgitation who underwent a mechanical mitral valve replacement at the age of 7 . Multiple chordae had ruptured on his anterior mitral valve leaflet and his posterior leaflet had eroded beyond hope of repair. He did well until the age of 25 when he presented with heart failure and had evidence of patient-prosthesis mismatch. He then underwent a redo sternotomy and a bioprosthetic mitral valve replacement and had an uneventful recovery. His yearly ECGs from the age of 25-32 revealed a superiorly directed p-wave morphology. He was asymptomatic during this time period with a very active lifestyle (sometimes $>40,000$ steps/day) (Figure 1). At the age of 32, he presented with vague symptoms of lightheadedness and was noted to be in atrial flutter (Figure 2).

He was taken for a catheter ablation of atrial flutter. Activation and entrainment mapping confirmed the rhythm to be typical, i.e. caval tricuspid isthmus dependent flutter. Qian et al studied atrial tachycardias and reported a high sensitivity and specificity ( 88 and $89 \%$ ) for a low right septal (coronary sinus ostium or tricuspid annular) site of origin for his pre-flutter $\mathrm{P}$ wave. ${ }^{3}$ Out of concern for possibly ablating his atrial pacemaker during caval tricuspid isthmus ablation, the decision was then made to cardiovert the patient and map the "sinus focus". Wide splits were noted contiguously in the posterior right atrium from the SVC to the IVC resulting in a line of conduction block. The site of earliest right atrial activation was noted to be sufficiently distant to the targeted line of CTI ablation. Atrial activation was noted to be primarily caudo-cranial (Figure 3, Panel A). CTI ablation was performed and bidirectional block was confirmed with differential atrial pacing. A new sinus rhythm map was created post-CTI ablation. Atrial activation postablation was now noted to be primarily cranio-caudal (Figure 3, Panel B) and the surface ECG now revealed inferiorly directed p-waves (Figure 4).

\section{Discussion:}

Normal p-wave morphology during sinus rhythm has been described as having an axis between 0 to +75 degrees. However, this is contingent upon an otherwise healthy atrium without conduction abnormalities. Our case highlights a young patient who has undergone two mitral valve surgeries, and as a result, two right atriotomies for cardiopulmonary bypass. During follow-up after the second surgery, his unusual p-wave axis was noted and it was unclear whether it was due to an atrial escape rhythm that had overtaken the sinus node or abnormal conduction of sinus rhythm. Electroanatomic activation mapping proved that he indeed had a sinus rhythm with conduction abnormality along the sites of his previous atriotomies. Additional ablation on the other side of the sinus node exit resulted in pseudo-normalization of his p-wave axis morphology. Four week follow up showed persistent inferiorly directed $\mathrm{P}$ wave, a finding in this patient consistent with persistent block across the caval tricuspid isthmus. 


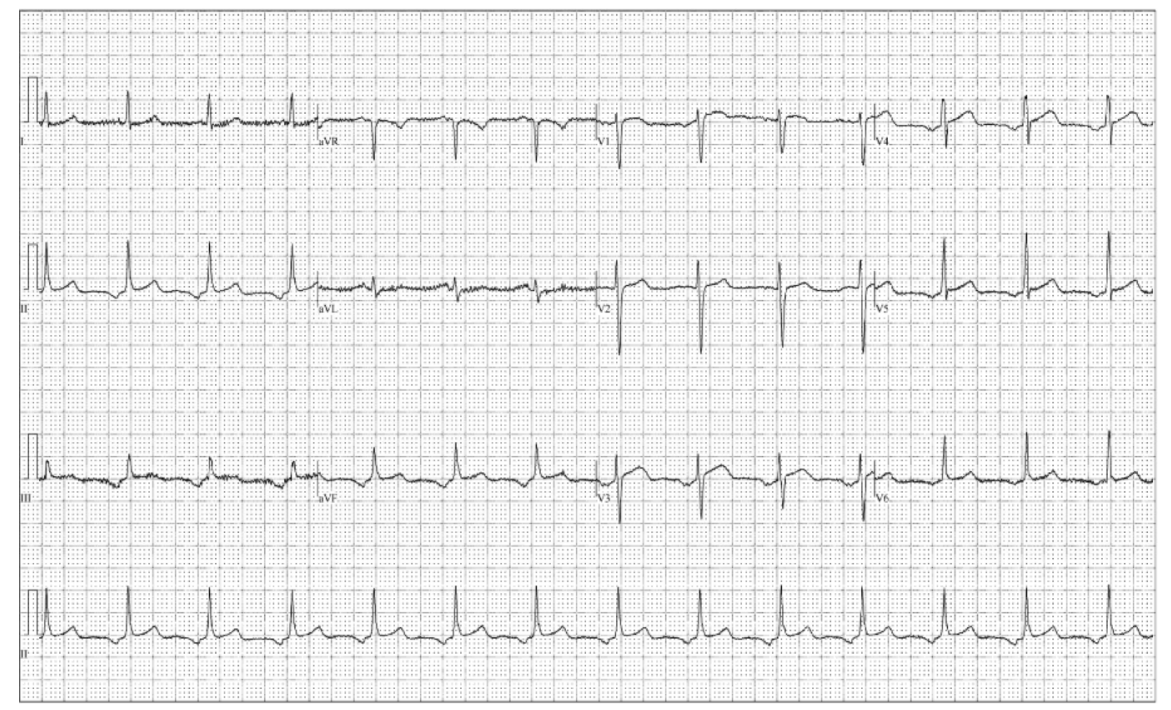

Figure 1: ECG after $2^{\text {nd }}$ MVR. Atrial rhythm with superiorly directed p-wave morphology.

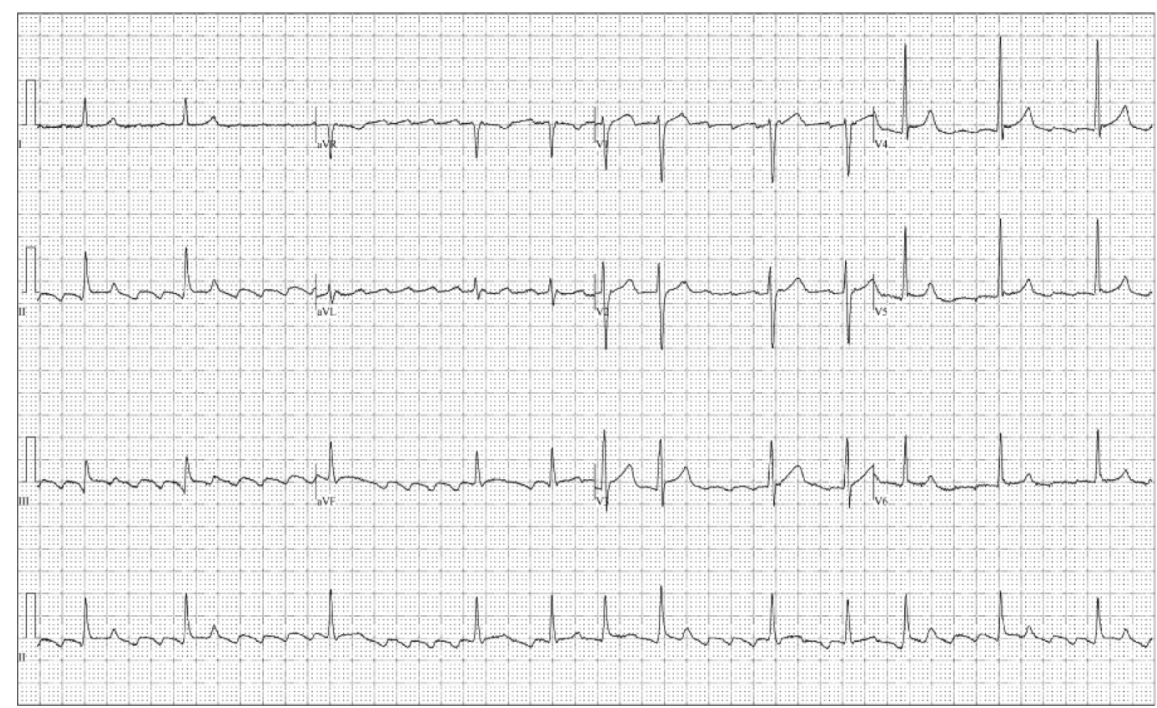

Figure 2: Typical atrial flutter with variable AV block.

\section{Hosted file}

image3.emf available at https://authorea.com/users/368495/articles/487594-normalization-of$\mathrm{p}$-wave-axis-after-cti-ablation

Figure 3: A - RAO (left), LAO (right), RAO Caudal (bottom) view of the right atrium with activation mapping during sinus rhythm prior to CTI ablation. B - RAO (left), LAO (right), RAO Caudal (bottom) view of the right atrium with activation mapping during sinus rhythm after CTI ablation. 


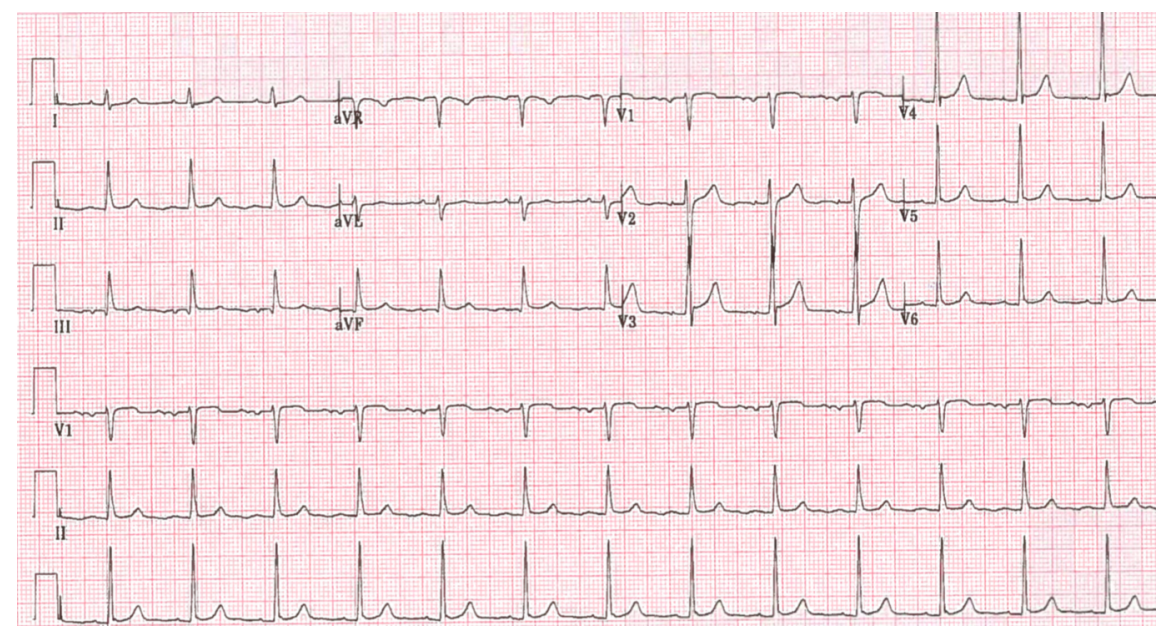

Figure 4: ECG after CTI ablation

\section{Discussion:}

1. O'Keefe JH. The complete guide to ECGs. 2nd ed. Physicians' Press; 2002:512 p.

2. Bo H, Heinzmann D, Grasshoff C, et al. ECG changes after percutaneous edge-to-edge mitral valve repair. Clin Cardiol. Nov 2019;42(11):1094-1099. doi:10.1002/clc.23258

3. Zhi-Yong Qian 1, Xiao-Feng Hou, Dong-Jie Xu, Bing Yang, Ming-Long Chen, Chun Chen, Feng-Xiang Zhang, Qi-Jun Shan, Ke-Jiang Cao, Jian-Gang Zou

An algorithm to predict the site of origin of focal atrial tachycardia.[Pacing Clin Electrophysiology. 2011] d. PMID: 21091746. DOI: 10.1111/j.1540-8159.2010.02980.x 